difference favours Oswald's (1986) healthy scepticism about the uniqueness of seasonal affective disorder.

Oswald, I. (1986) What is seasonal affective disorder? British Medical Journal, 292, 1326.

VON KNORRING, L. (1975) The experience of pain in depressed patients. Neuropsychobiology, 1, 155-165.

Newcastle Hospital

Brian O'SHEA

Greystones

County Wicklow

Ireland

\section{The rarity of mania in Down's syndrome}

SIR: Drs Cooper \& Collacott (BJP, June 1993, 162, 739-743) reviewed seven case reports of mania in people with Down's syndrome. The authors suggest that the rarity of mania in these patients, especially women, might be due to unique biochemical characteristics of this syndrome, perhaps with gender differences.

One problem with this interpretation is the lack of validated diagnostic criteria and assessment instruments for people with mental retardation (Campbell \& Malone, 1991; Sturmey, 1993). Impaired language and intellect can make it difficult to assess symptoms in these patients, and there is a tendency to rely on subjective interpretations of behavioural observations to make psychiatric diagnoses. Indeed, behaviour problems are a common reason for psychiatric referral of these patients (Aman, 1987; Howland, 1992). Behaviour problems often are simply attributed to the mental retardation, or are loosely diagnosed as an organic brain syndrome, rather than recognised as a possible manifestation of an underlying comorbid psychiatric disorder. Behaviour problems can also mask other symptoms. This, together with the difficulty of assessing cognitive symptoms due to impaired language and intellect, can lead to a failure to diagnose specific psychiatric disorders (Howland, 1992). In addition, the known association of Down's syndrome with the early onset of Alzheimer's disease might also bias doctors to attribute any psychiatric or behavioural symptoms to the early stages of dementia.
A second problem is the confounding effect of treatment on the recognition of psychiatric symptoms in people with mental retardation. Neuroleptics, anticonvulsants, and sedatives are the most commonly used drugs in these patients, typically to control behaviour problems (Aman, 1987). These drugs have antimanic effects, and their widespread but non-specific use to treat behaviour problems might thus lead to a spuriously low prevalence of mania. The confounding effect of anticonvulsant treatment has been similarly suggested to explain the rarity of mania in some patients with epilepsy (Howland, 1993).

These diagnostic and treatment issues provide an alternative and, I believe, more likely explanation for the rarity of mania in Down's syndrome. Future research investigating the clinical and biological aspects of mania and other psychiatric disorders in people with Down's syndrome should carefully control for these important confounding factors.

Aman, M. G. (1987) Overview of pharmacotherapy: current status and future directions. Journal of Mental Deficiency Research, 31, $121-130$.

Campbell. M. \& Malone, R. P. (1991) Mental retardation and psychiatric disorders. Hospital and Community Psychiatry, 42, 374-379.

Howland, R. H. (1992) Fluoxetine treatment of depression in mentally retarded adults. Journal of Nervous and Mental Disease. 180, 202-205.

(1993) Bipolar disorder associated with primary generalised epilepsy. British Journal of Psychiatry, 162, 699-700.

StURMEY, P. (1993) The use of DSM and ICD diagnostic criteria in people with mental retardation. Journal of Nervous and Mental Disease, 181, 38-41.

ROBERT H. HOWLAND

Western Psychiatric Institute and Clinic

University of Pittsburgh School of Medicine.

Pittsburgh

Pennsylvania 15213

$U S A$

\section{CORRIGENDUM}

$B J P$, January 1994, 164, 128. "Benzodiazepine fatal poisonings": the third sentence should read "We were surprised by the high number of benzodiazepines not only involved in attempted suicide $(46.3 \%$ of all drugs taken, single or in combination with other drugs) but also in completed suicide $(43.8 \%)$ ". 